\title{
Ortogeriatria: un nuovo modello di assistenza ai pazienti anziani con frattura di femore e comorbilità
}

\section{Comprehensive care of elderly patients with hip fracture: the orthogeriatric model}

\author{
Carlo Frondini *, Maria L. Lunardelli \\ UO Geriatria "Lunardelli", Azienda Ospedaliero-Universitaria "Sant'Orsola-Malpighi", Bologna
}

Ricevuto il 31 luglio 2009; accettato il 28 settembre 2009

disponibile online il 18 aprile 2010

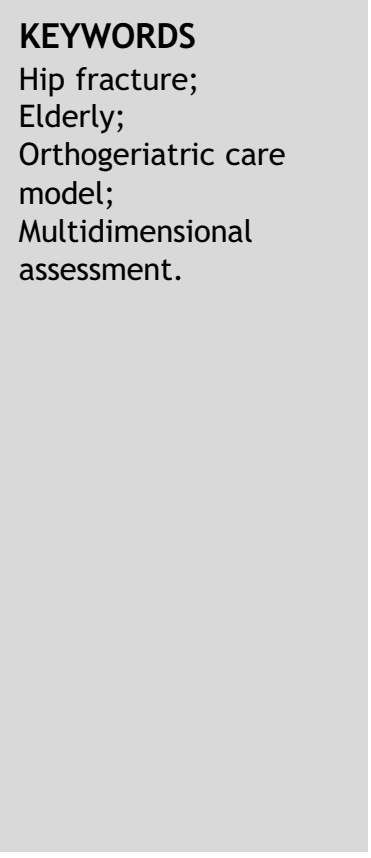

\begin{abstract}
Summary
Introduction: Hip fractures in the elderly are a major source of morbidity and mortality. Interdisciplinary hospital care models proposed for the treatment of these patients include consultant teams, integrated orthopedic-geriatric care, and comprehensive geriatric-led care settings. A prospective interventional cohort study was conducted in 4 public hospitals in the Emilia-Romagna Region of Italy to compare the outcomes of these different care models. This report presents the preliminary results obtained with an orthogeriatric model in one of these centers, a large teaching hospital in Bologna.

Materials and methods: Beginning in February 2008, all patients older than 75 years admitted to the University of Bologna's Sant'Orsola-Malpighi Hospital for hip fractures were cared for in an orthogeriatric unit. The unit consisted of 10 beds in the orthopedic ward that were managed by a geriatric specialist and a multidisciplinary team, which met daily and included an orthopedic surgeon, a physiatrist, a nurse case-manager, staff nurses, a physical therapist, and a social worker. The management protocol included a thorough geriatric work-up to identify comorbidities and risk factors, systematic assessment and prevention of pain and acute disorientation, early verticalization and moblization, postacute rehabilitation therapy, family support, and regular follow-up after discharge. Preliminary results were compared with those achieved in the same orthopedic ward prior to the creation of the Orthogeriatric Unit.

Results: During 2008, 226 elderly patients (mean age $86.2 \pm 5.5$ years), $73.4 \%$ of whom were women, were admitted to the Orthogeriatric Unit for hip fractures. The mean Charlson comorbidity index of this cohort was $3.0 \pm 1.8$ ). Half the patients had Activity of Daily Living scores $\leq 4$, and cognitive impairment was common (mean score on Short Portable Mental Status
\end{abstract}

\footnotetext{
* Corrispondenza: UO Geriatria "Lunardelli", Azienda Ospedaliero-Universitaria "Sant'Orsola-Malpighi”, via Albertoni 15 - 40138 Bologna.
} E-mail: carlo.frondini@aosp.bo.it (C. Frondini). 
Questionnaire: $5.9 \pm 3.2$ ). Compared with figures obtained in the hospital's orthopedic ward prior to 2008 , in-hospital mortality dropped from $5.98 \%$ to $3.98 \%$. The mean overall length of hospitalization was not significantly reduced, but the mean stay in the Orthogeriatric unit decreased by almost 2 days (to 10.46 days from 12.44 days in the traditional orthopedic unit). Discussion: Compared with traditional care models, the orthogeriatric model may allow better integration of the health-care resources available for the management of hip fractures in the elderly, and this improvement may have positive effects on the outcome of these cases.

(c) 2010 Elsevier Srl. All rights reserved.

\section{Introduzione}

La frattura di femore è un problema sanitario di grande impatto sull'ospedale e sui servizi territoriali, destinato a crescere con l'aumento della popolazione anziana.

In Italia si verificano ogni anno oltre 86.000 fratture di femore, con un costo complessivo superiore a 1 miliardo di euro (di cui 394 milioni per costi relativi all'ospedalizzazione, i rimanenti per i costi di riabilitazione e i costi sociali) [1].

Anche se la mortalità perioperatoria si è notevolmente ridotta negli ultimi anni, la mortalità permane molto elevata sia a un mese dall'intervento (5-10\%) sia a un anno (intorno al $30 \%$ ) [2]; inoltre, la perdita di autonomia dopo una frattura può arrivare fino al $60 \%$, con un elevato tasso di istituzionalizzazione (20-25\%).

Le ricerche epidemiologiche supportano l'idea che per migliorare gli outcome sia necessario un approccio - radicalmente diverso da quello tradizionale - basato sulla gestione integrata di competenze ortopediche, geriatriche e riabilitative [3-6].

In ambito ospedaliero, i percorsi assistenziali si caratterizzano per un coinvolgimento diverso del geriatra: dalla consulenza programmata a modelli di gestione coordinata all'interno del reparto di Ortopedia, fino alla creazione di una struttura ortogeriatrica dedicata [7].

A prescindere dalle modalità organizzative, secondo il National service framework for older people inglese, ogni ospedale per acuti dovrebbe dotarsi di almeno un centro di eccellenza di Ortogeriatria [8].

Recenti review Cochrane non consentono di individuare un modello gestionale di riferimento per quanto riguarda l'efficacia e per trarre conclusioni definitve sono ritenuti necessari studi di confronto fra esperienze differenti, con omogeneità di raccolta dei dati [9].

\section{Il progetto "Ortogeriatria" della Regione Emilia-Romagna}

Nel 2006, in Emilia-Romagna, le dimissioni ospedaliere per fratture di femore in soggetti di età $>75$ anni sono state oltre 6.000 (banca dati Schede di Dimissioni Ospedaliere). Le linee strategiche del governo clinico della frattura di femore nell'anziano proprie del Sistema Sanitario Regionale sono: elevati standard di qualità, contenimento dei costi e facilitazione dei percorsi di continuità tra ospedale e territorio (Piano Sanitario e Sociale 2007-2009).

In questo contesto, alcune realtà geriatriche della regione, che già si occupavano della gestione dell'anziano fratturato in collaborazione con gli ortopedici, hanno promosso un progetto di ricerca per confrontare diversi modelli ortogeriatrici, al fine di ottenere informazioni sugli aspetti in grado di incidere maggiormente sugli outcome. Tale progetto è stato approvato e finanziato come Ricerca RegioneUniversità Area 2 Governo Clinico 2007-2009 e ha preso avvio nei primi mesi del 2008.

La ricerca denominata Modelli per la gestione clinica integrata e per la continuità assistenziale degli anziani con frattura di femore è stata approvata dal comitato etico dell'Azienda Ospedaliero-Universitaria di Bologna, che è coinvolta come capofila e coordina le altre unità partecipanti al progetto. Con riferimento al tipo di integrazione tra il geriatra e l'ortopedico, vengono indagati i seguenti quattro modelli di intervento:

- consulenza geriatrica programmata 5 volte la settimana nel reparto di Ortopedia (Parma);

- gestione geriatrica all'interno del reparto di Ortopedia (co-management) con responsabilità differenziate (Reggio Emilia);

- sezione dedicata di Ortogeriatria nell'ambito dell'Unità Operativa di Ortopedia (Bologna);

- reparto di Postacuzie precoce integrato con servizio di telemedicina dopo il rientro a domicilio (Ferrara).

In tutti i centri viene effettuata la valutazione di un "core set" di dati, raccolto con software elaborato ad hoc. I parametri sono suddivisibili in quattro gruppi.

1. Caratteristiche basali dei pazienti (età, sesso, provenienza e convivenza, supporto in atto e uso dei servizi); tipo di frattura, tipo di caduta, abilità funzionali prefrattura (Activities of Daily Living, ADL; Instrumental Activities of Daily Living, IADL), deambulazione; indice di Charlson, terapia farmacologica a domicilio, scala di Conley, Short Portable Mental Status Questionnaire (SPMSQ), Geriatric Depression Scale (GDS), severità dello stato clinico (score APACHE), parametri biochimici all'ingresso (albuminemia, paratormone, vitamina D 25-OH, proteina C-reattiva, emoglobina).

2. Complicanze intraospedaliere: cardiovascolari maggiori e sistemiche, ortopediche, incidenza di delirium, lesioni da pressione, infezioni.

3. Parametri relativi al modello organizzativo generale: tempo di attesa preintervento, tipo di intervento, tempo di verticalizzazione, durata della degenza per setting di cura.

4. Parametri relativi al follow-up: luogo di dimissione, sede e durata della fase riabilitativa, abilità funzionali per ADL, deambulazione, salire e scendere le scale; utilizzo di servizi territoriali; eventuale istituzionalizzazione; data dell'eventuale decesso. Il follow-up è previsto a 3 , 6 e 12 mesi mediante intervista telefonica con apposita scheda di rilevazione.

I primi risultati della ricerca regionale saranno disponibili nell'autunno 2009. Il follow-up sarà completato nel 2010. 
Gli autori dichiarano che lo studio presentato è stato realizzato in accordo con gli standard etici stabiliti nella Dichiarazione di Helsinki, e che il consenso informato è stato ottenuto da tutti i partecipanti prima del loro arruolamento allo studio.

\section{L'esperienza di Bologna}

Nel 2006, presso l'Azienda Ospedaliero-Universitaria "Sant'Orsola-Malpighi" sono stati ricoverati 386 pazienti con frattura di femore, di cui $330(85,49 \%)$ di età > 75 anni, con prevalenza del sesso femminile $(76 \%)$. La maggior parte di questi pazienti era affetta da malattie croniche complesse, con elevato rischio di complicanze in conseguenza della frattura (immobilità, disidratazione, stress acuto, ipovolemia ecc).

Scopo del progetto "Ortogeriatria" della suddetta Azienda è delineare il percorso assistenziale dell'anziano con frattura di femore attraverso un approccio integrato multidisciplinare e multiprofessionale (ortopedico, geriatrico, riabilitativo, di nursing e sociale), con l'adozione di pratiche terapeutico-assistenziali definite in un modello di continuità assistenziale.

A partire dal febbraio 2008, nell'ambito della UO di Ortopedia e Traumatologia (direttore: dott. M. Laus) sono stati individuati 10 posti letto di Ortogeriatria, riservati ai pazienti di età $>75$ anni con frattura di femore e comorbilità. Responsabile della gestione clinico-geriatrica è il dott.

C. Frondini della UO Geriatria "Lunardelli". Punti qualificanti dell'Ortogeriatria sono:

- la valutazione clinico-geriatrica precoce e accurata, al fine di stabilizzare i problemi attivi prima dell'intervento;

- la valutazione sistematica e la prevenzione/il trattamento del dolore;

- la valutazione sistematica e la prevenzione/il trattamento degli stati confusionali acuti;

- la valutazione multidimensionale geriatrica, in particolare dell'autonomia prefrattura e del supporto sociofamiliare del paziente, al fine di elaborare fin dai primi giorni di ricovero un coerente progetto riabilitativo;

- la prevenzione e la gestione delle complicanze mediche nel postoperatorio;

- la precoce mobilizzazione e "verticalizzazione" del paziente;

- la gestione in team con incontri, sia strutturati periodici sia informali quotidiani, tra le varie figure professionali per la rivalutazione clinico-assistenziale del caso e la pianificazione della dimissione o del trasferimento;

- la prosecuzione in fase postacuta del trattamento riabilitativo in Medicina Fisica e Riabilitazione ovvero (nei casi con minore potenzialità di recupero per comorbilità o deterioramento cognitivo) presso la sezione Post Acuti Riabilitazione Estensiva (PARE) Geriatria "Lunardelli";

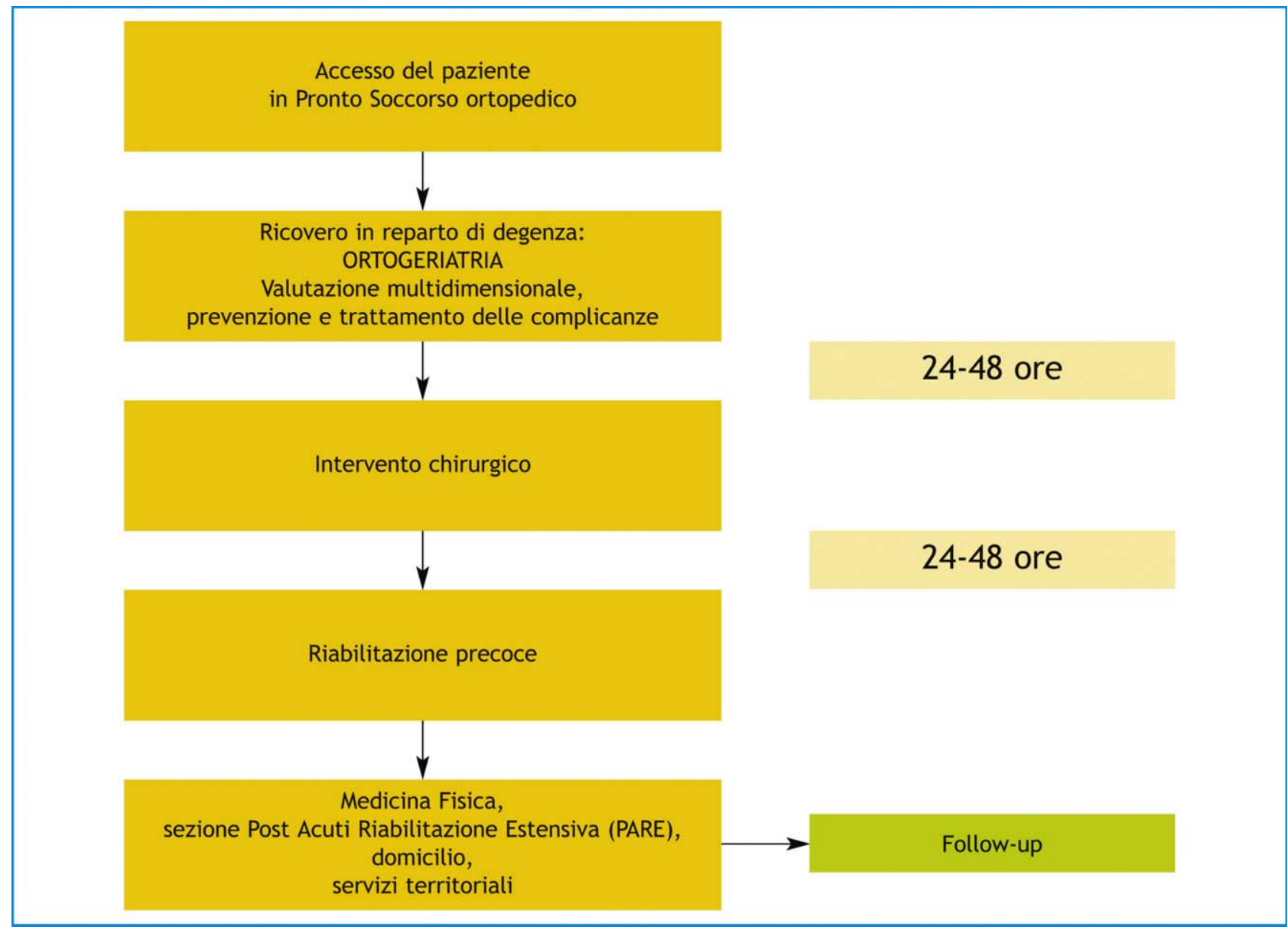

Figura 1 Percorso assistenziale del paziente con frattura di femore nella fase ospedaliera. 


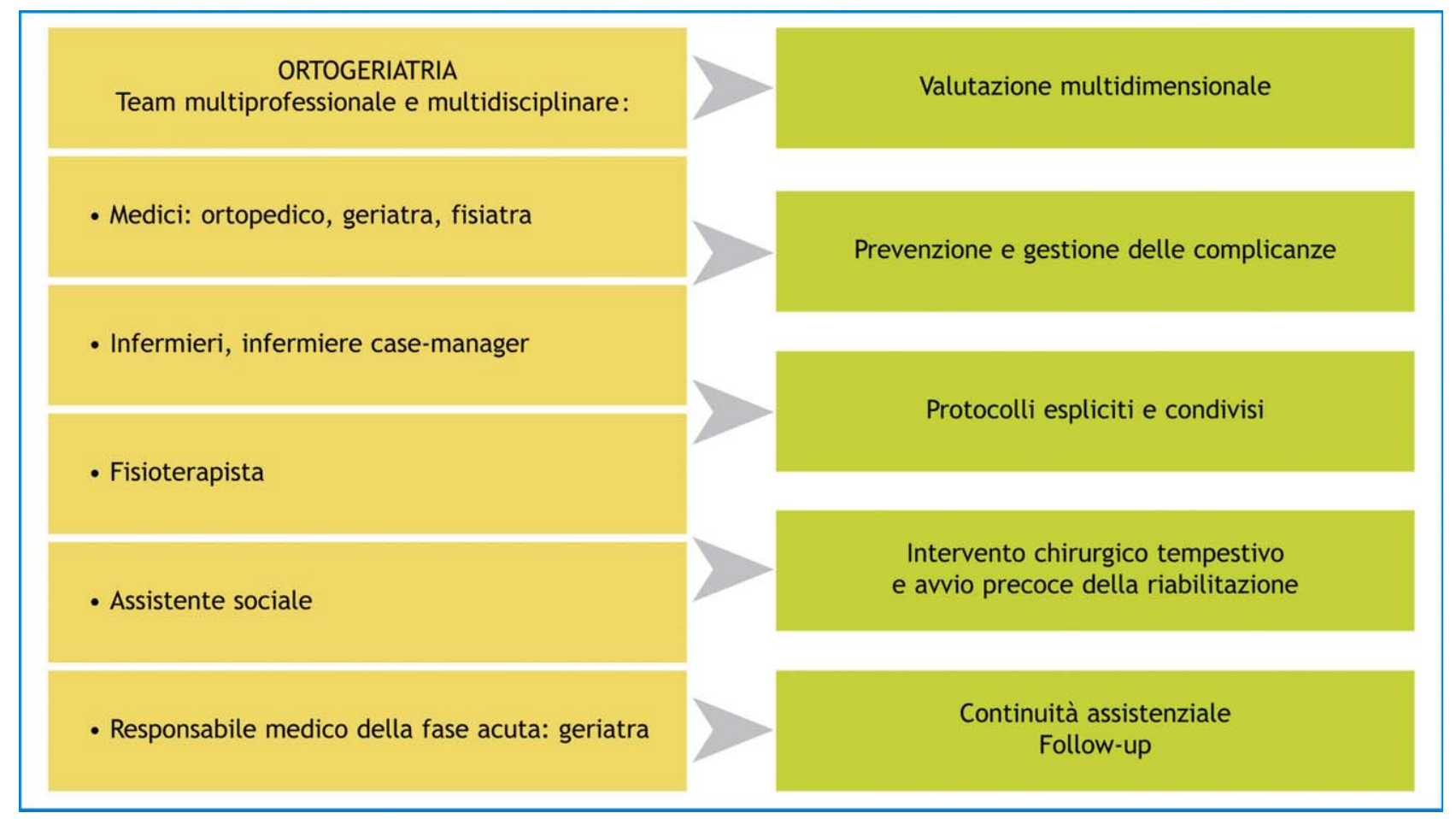

Figura 2 Modello organizzativo-assistenziale in Ortogeriatria.

- in presenza di buone condizioni cliniche e di un adeguato supporto familiare, la dimissione al domicilio con attivazione della continuità assistenziale, mediante segnalazione tempestiva ai servizi del territorio per proseguire la riabilitazione al domicilio o in ambulatorio;

- il follow-up con cadenze periodiche finalizzato alla valutazione del recupero funzionale e alla valutazione/prevenzione del rischio di caduta e di ri-frattura.

\section{Il percorso assistenziale}

La presa in carico del paziente con frattura di femore nella fase ospedaliera può essere così riassunta ( fig. 1):

- inquadramento diagnostico in Pronto Soccorso ortopedico;

- ricovero in Ortogeriatria per intervento chirurgico precoce, valutazione multidimensionale e precoce mobilizzazione/ riabilitazione;

- prosecuzione, in fase postacuta, del trattamento riabilitativo in Medicina Fisica ovvero presso la sezione PARE;

- dimissione strutturata con eventuale fase riabilitativa postospedaliera;

- follow-up.

\section{Il modello organizzativo-assistenziale}

Centrale, nel modello ortogeriatrico, è il team multiprofessionale e multidisciplinare. La scelta è motivata dalla necessità di porre il paziente al centro dell'assistenza e di integrare i diversi apporti professionali in un progetto coordinato di presa in carico del malato in ogni fase del percorso assistenziale ( fig. 2). Poiché gli anziani con frattura hanno comorbilità associate e una diminuita riserva funzionale, necessitano di una gestione clinica quotidiana atta a prevenire le complicanze responsabili di ritardi nel trattamento chirurgico e di aumento della mortalità nel periodo peri e postoperatorio.

Il team è formato dall'ortopedico, dal geriatra, dal fisiatra, dall'infermiere case-manager, dagli infermieri, dal fisioterapista e dall'assistente sociale. L'interazione tra i componenti del team è quotidiana; gli incontri programmati sono previsti in fase preoperatoria, nell'immediato postoperatorio e poi almeno settimanalmente, con lo scopo di gestire collegialmente il programma terapeutico e di verificare in itinere e a breve termine la validità e/o l'appropriatezza degli interventi intrapresi (fig. 3).

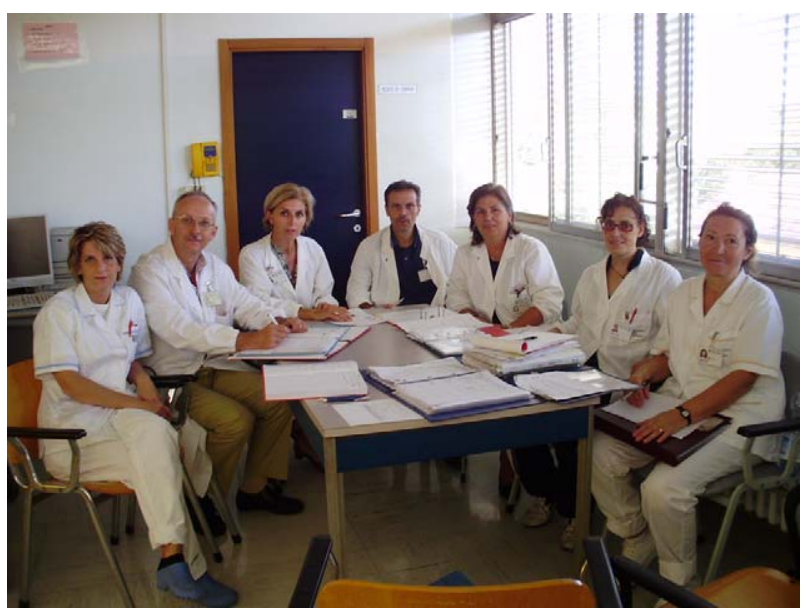

Figura 3 Riunione del team ortogeriatrico: infermiere case-manager, geriatri, ortopedico, fisiatra, assistente sociale, fisioterapista. 
Tabella 1 Indicatori in Ortogeriatria.

\begin{tabular}{lcc}
\hline Anno di riferimento & 2007 (Ortopedia) & 2008 (Ortogeriatria) \\
\hline Degenza media in Ortopedia/Ortogeriatria (giorni) & 12,44 & 10,46 \\
\hline Degenza media totale (giorni) & 23,48 & 25,2 \\
\hline Mortalità totale ospedaliera (\%) & 5,98 & 3,98 \\
\hline
\end{tabular}

Responsabile della gestione assistenziale del paziente dall'ammissione alla dimissione dal reparto è l'infermiere case-manager, che garantisce il coordinamento e l'erogazione di un'assistenza complessiva ad alta personalizzazione e la continuità del percorso assistenziale. Inoltre, insieme con l'assistente sociale, costituisce un riferimento stabile per il paziente e la famiglia.

La presenza del fisioterapista dedicato mira a garantire la presa in carico tempestiva del paziente (entro 24 ore dall'intervento), a favorire il precoce avvio della fase di recupero, a facilitare la preparazione e l'accesso al percorso riabilitativo personalizzato che il paziente svolgerà successivamente in ambiente riabilitativo o al domicilio.

\section{La valutazione degli indicatori}

L'attivazione del nuovo modello assistenziale ortogeriatrico è contestuale al progetto Ricerca Regione-Università Area 2 Governo Clinico 2007-2009 di cui l'Azienda OspedalieroUniversitaria "Sant'Orsola-Malpighi" di Bologna è capofila. L'impatto clinico-organizzativo del percorso e il grado di implementazione del progetto rispetto ai risultati attesi verranno monitorati attraverso un protocollo di raccolta dei dati comprensivo di indicatori di processo, di esito e clinico-organizzativi.

\section{Risultati preliminari}

Nel corso del 2008 sono stati ricoverati in Ortogeriatria 226 pazienti anziani (età media $86,2 \pm 5,5$ anni), di cui il $73,4 \%$ donne, con frattura di femore e comorbilità (indice di Charlson 3,0 $\pm 1,8)$.

I pazienti presentavano un elevato consumo di farmaci (in media 4,54 $\pm 2,79$ farmaci al domicilio), una ridotta autonomia nelle attività funzionali (ADL basali $\leq 4$ nel $50 \%$ dei casi), un'elevata prevalenza di deterioramento cognitivo (score SPMSQ 5,9 \pm 3,2).

I dati del primo anno di attività sono incoraggianti in termini sia di riduzione della degenza media in Ortopedia/ Ortogeriatria sia di mortalità ospedaliera, anche se non si è osservata una riduzione della durata totale dell'ospedalizzazione (tabella 1).

Altrettanto importanti appaiono gli aspetti clinici di riduzione dell'incidenza di ulcere da pressione e l'implementazione del progetto Ospedale senza Dolore (passaggio dalla terapia al bisogno alla terapia programmata), così come l'attivazione della dimissione con riabiltazione al domicilio (continuità ospedale-territorio) in precedenza non attuata dall'Azienda Ospedaliero-Universitaria "Sant'Orsola-Malpighi" di Bologna. L'implementazione di quest'ultimo aspetto potrà consentire, in futuro, una minore durata della degenza ospedaliera.

Il modello ortogeriatrico consente, inoltre, la raccolta sistematica di parametri biologici e funzionali che influenzano gli outcome; in tutti i pazienti sono valutati gli indicatori del rischio di caduta, la presenza in anamnesi di osteoporosi, il trattamento farmacologico domiciliare e i livelli di vitamina D 25-OH, paratormone, calcemia, albuminemia all'ingresso in ospedale.

Nei pazienti anziani con frattura è stata evidenziata una vera e propria "epidemia" di severa ipovitaminosi D e iperparatiroidismo secondario. Inoltre i dati confermano la sottostima dell'osteoporosi e l'inadeguatezza del trattamento negli anziani che si fratturano, già segnalata in letteratura [10-12].

L'intervento del geriatra può contribuire a ridurre e/o sospendere farmaci potenzialmente pericolosi, implementando per contro l'uso di farmaci di provata utilità e a basso costo come la vitamina $D$ non idrossilata, al fine di ridurre il rischio di cadute e di ri-fratture in questa popolazione realmente "fragile".

\section{Conflitto di interesse}

Gli autori dichiarano di essere esenti da conflitto di interessi.

\section{Bibliografia}

[1] Piscitelli P, Iolascon G, Gimigliano F, Muratore M, Camboa P, Borgia $\mathrm{O}$, et al., SIOMMMS study group, CERSUM research group. Incidence and costs of hip fractures compared to acute myocardial infarction in the Italian population: a 4-year survey. Osteoporos Int 2007;18(2):211-9.

[2] Parker M, Johansen A. Hip fracture. BMJ 2006;333(7557): 27-30.

[3] Adunsky A, Arad M, Levi R, Blankstein A, Zeilig G, Mizrachi E. Five-year experience with the "Sheba" model of comprehensive orthogeriatric care for elderly hip fracture patients. Disabil Rehabil 2005;27(18-19):1123-7.

[4] Barone A, Giusti A, Pizzonia M, Razzano M, Palummeri E, Pioli G. A comprehensive geriatric intervention reduces short- and longterm mortality in older people with hip fracture. J Am Geriatr Soc 2006;54(4):711-2.

[5] Antonelli Incalzi R, Gemma A, Capparella O. Orthogeriatric Unit: a thinking process and a working model. Aging Clin Exp Res 2008;20(2):109-12.

[6] Management of hip fracture in older people. SIGN Publication 2009 June; 111. http://www.sign.ac.uk/pdf/sign111.pdf.

[7] Pioli G, Giusti A, Barone A. Orthogeriatric care for the elderly with hip fractures: where are we? Aging Clin Exp Res 2008;20(2): 113-22.

[8] Department of Health. National service framework for older people. 2001. http://www.dh.gov.uk/en/publicationsandstatistics/ publications/publicationspolicyandguidance/DH_4003066. 
[9] Cameron ID, Handoll HH, Finnegan TP, Madhok R, Langhorne P. Co-ordinated multidisciplinary approaches for inpatient rehabilitation of older patients with proximal femoral fractures. Cochrane Database Syst Rev 2001;(3):CD000106.

[10] Lips P. Vitamin D deficiency and secondary hyperparathyroidism in the elderly: consequences for bone loss and fractures and therapeutic implications. Endocr Rev 2001;22(4): 477-501.
[11] Giusti A, Barone A, Razzano M, Pizzonia M, Oliveri M, PalummeriE, et al. High prevalence of secondary hyperparathyroidism due to hypovitaminosis $D$ in hospitalized elderly with and without hip fracture. J Endocrinol Invest 2006;29(9):809-13.

[12] Frondini C, Lucicesare A, Cerchi N, Lunardelli ML. Diagnosi di osteoporosi, ipovitaminosi D ed appropriatezza del trattamento nei pazienti anziani con frattura di femore: esperienze dell'Ortogeriatria di Bologna. Atti VII Congresso Nazionale GIBIS. 2009. 